\section{Library-to-library screening}

Conventional protein-protein interaction screens are based on binding a protein library to a single bait protein. On page 683 , Pelletier et al. describe a way of greatly increasing the number of combinations sampled by screening one protein library against another. Their method, which is derived from an existing screen for reconstituting DHFR enzymatic activity from two fragments, allowed them to uncover sequence biases important for binding, and to identify a new leucine zipper pair.

\section{African rice virus overcome?}

Rice yellow mottle virus (RYMV) is responsible for devastating losses in African rice production - and incidence of the disease is on the rise. Conventional approaches for breeding RYMV-resistant cultivars have proved impractical, but in this issue, Pinto et al. (p. 702) describe an effective transgenic approach. Using the concept of pathogen-derived resistance, they introduced transgenes encoding fragments of the RYMV genome, producing transgenic lines with a high degree of resistance to RYMV.

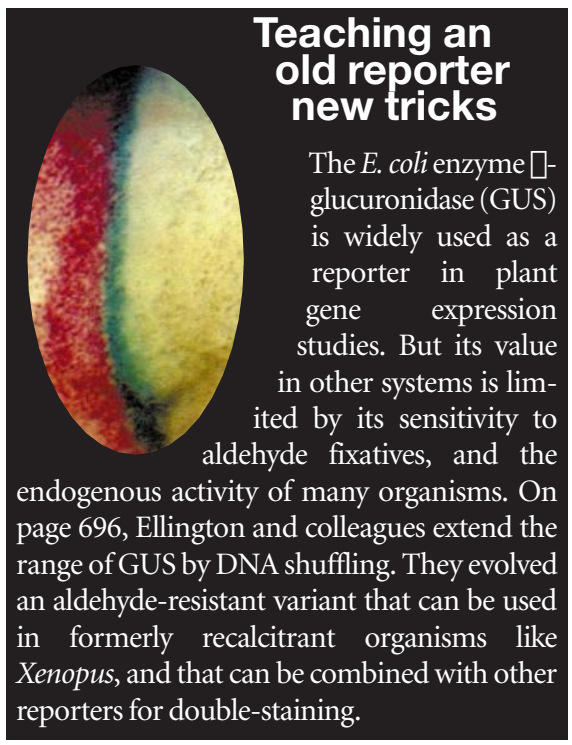

\section{Sugar-free spuds}

Potatoes are typically stored in the cold to prevent sprouting. But this can cause starch to break down into hexoses - ending in culinary disasters like sweetened mashed potatoes. Rausch and colleagues (p. 708) have come to the rescue by engineering potato plants that overexpress an invertase inhibitor. This inhibits the cold-induced sweetening by $75 \%$ without affecting potato quality.

Research Briefs written by Natalie DeWitt.

\title{
Engineering neurons to treat Parkinson's disease
}

Transplantation of embryonic neurons can restore functional dopaminergic neurons in the brains of patients with Parkinson's disease. But while promising, cell transplantation therapy is still out of reach to the vast majority of patients, in part because of the inaccessibility of human embryonic tissue. On page 653, Arenas and colleagues describe a method for engineering virtually unlimited quantities of dopaminergic neurons needed for transplantation

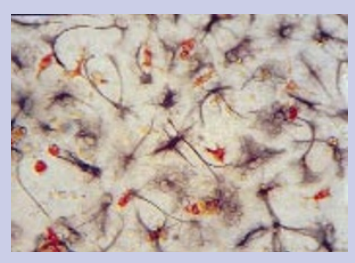
studies to treat Parkinson's disease. The authors first obtained neuronal stem cells from mouse cells transfected with a transcription factor that encourages cells to adopt a neuronal fate. They then co-cultured the cells with astrocytes, which release a factor that induces development into dopaminergic neurons. The engineered cells released dopamine, and some maintained the characteristics of dopaminergic neurons for up to two weeks after implantation into mouse brains.

\section{Proteomics get complex}

The biochemist's penchant for grinding and fractionating can have the unfortunate consequence of destroying protein complexes-ultimately distorting our view of how proteins are organized and regulated in the cell. On page 676, Link et al. describe a new proteomics tool that alleviates this problem by making it possible to identify each component of large macromolecular complexes containing more than 100 proteins. Their system combines multidimensional liquid chromatography and tandem mass spectrometry to separate and fragment peptides with an algorithm to infer amino acid sequences from peptide masses.

\section{Anti-inflammatory antibodies}

Therapeutic monoclonal antibodies against the pro-inflammatory cytokine TNF- $\alpha$ have been successfully used to treat chronic inflammatory diseases. In this issue, Mouritsen and colleagues (p. 666) take this approach one step further by developing a method to immunize against self TNF- $\alpha$. Mice immunized with modified TNF- $\alpha$ stimulated a booster-dependent T-cell dependent anti-TNF- $\alpha$ response-relieving symptoms of cachexia and arthritis.

\section{Efficient antigen presentation}

On page 670, Lunde et al. describe a technology that could eventually help in vaccine design by boosting $\sim 1000$-fold the efficiency of antigen presentation to Tcells. The technology is based on the relationship between the strength of T-cell responses and the number of antigen Molecules processed and delivered to antigen presenting cells (APC) such as Bcells. Lunde and colleagues enhanced this process by designing a recombinant antibody to which APC targeting sequences were added to improve delivery, and T-cell epitopes were added to stimulate Tcells.

\section{Weed whacker}

Herbicide-resistant crops are an important weapon in the farmer's arsenal against invasive weeds. On page 712 Hussey and colleagues describe genetic engineering of plants resistant to dinitroaniline herbicides, which are effective against an especially aggressive class of weeds. The method relies on overexpression of a mutant tubulin isoform, which the authors show does not interfere with the generation and propagation of the transgenic plants.

\section{Illuminating protein folding}

In this issue, Waldo et al. (p. 691) describe a

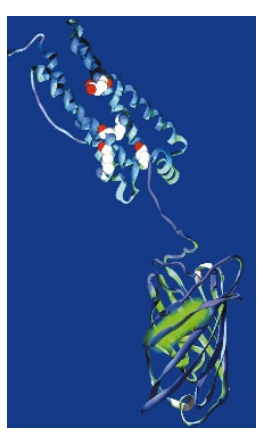
way to rapidly select protein variants capable of better folding in $E$. coli. This method is based on the surprising observation that, when fused to the $\mathrm{C}$ terminus of other proteins, GFP fluorescence is sensitive to folding of the upstream fusion partner, which is in turn related to the folding of the upstream protein alone. The GFP signal therefore can be used a marker to identify robustly folding protein variants.

\section{Peptide mimicry for cancer vaccines?}

Tumor-associated carbohydrate (TAC) antigens are prime candidates for cancer vaccines because of their involvement in metastasis and the rapid growth of tumors. But their inability to elicit a T-cell dependent immune response often makes them weak immunogens. Kieber-Emmons and colleagues (p. 660) have overcome this obstacle by designing a peptide that mimics a TAC antigen. Vaccination of mice with the peptide mimotope reduced tumor growth and prolonged survival. 